\title{
Customer Service Management for Grid Monitoring and Accounting Data
}

\author{
Timo Baur ${ }^{1}$ and Samah Bel Haj Saad ${ }^{2}$ \\ ${ }^{1}$ Munich Network Management Team \\ Leibniz Rechenzentrum Garching \\ Boltzmannstr. 1 \\ D-85748 Garching \\ ${ }^{2}$ Munich Network Management Team \\ Universität der Bundeswehr München \\ Werner-Heisenberg-Weg 39 \\ D-85579 Neubiberg
}

\begin{abstract}
Experiences with the management of Grid specific monitoring and accounting data have shown that current approaches do not sufficiently support a distinction between providers, users and customers of a Grid. This gap can be filled by the use of Customer Service Management techniques which enable customers to individually monitor and control their subscribed services. We adapt a Customer Service Management scenario to Grid environments and outline an architecture dedicated to the management and visualization of monitoring and accounting data. To proof the concept, a prototype based on standard Grid components which manages user's needs and interactions with the resource provider is presented.
\end{abstract}

\section{Introduction}

Customer oriented services have become a strategic success factor of the IT industry. In the past, methods for Customer Service Management (CSM) helped to establish customer-oriented interfaces between internet service providers and customers, which enable a logical view and management of a customer's subscribed services. With the increasing interest of science and business in Grids, the integration of these methods within Grid infrastructures now become an important challenge.

A CSM interface offers the possibility to get current and appropriate information about the condition of services [1, e.g. a monitoring or an accounting service. This enables a customer to ask for internal processes and service states at the providers sites that are related to the provisioning of his subscribed resources and services (e.g. maintenance times).

In this paper, we discuss how methods of CSM can be adapted to a Grid environment in order to support customer needs and to inform and notify them about service states and relevant data. As a concrete example, we develop a concept based on CSM to visualize monitoring and accounting relevant data as a customer specific service for VOs in a Grid environment. 
This paper is organized as follows: In section 2 we discuss related work and the state of the art of CSM in Grid environments. Moreover, in section 3, we introduce monitoring and accounting services as a use case. In section 4 a generic CSM scenario is applied to a Grid. We provide a description of CSM functionalities and indicate, where an adaptation or usage of Grid concepts and components can be applied. Finally in section [5 we address the implementation of a prototype that gathers monitoring and accounting information for specific Grid customers.

\section{State of the Art}

Currently, many companies and research institutions are developing and running CSM tools to manage their networks, among others the CSM system of the German Research Network (DFN) and the pan-european research and education backbone Geant2. In the area of Grid systems, where service and customer 2] orientation as well as QoS and SLA integration [3, 4, [5] are still challenging issues, CSM tools have not been applied yet. Many tools, e.g., MapCenter [6], Ganglia 7] or MonALISA [8] have been designed to cope with new Grid monitoring challenges, but most work is provider centric and does not take into account the requirements of customer-orientation.

CSM extends the provider's service management towards the customers and enables them to monitor and control up-to-date and meaningful information about service specific QoS parameters [9]. Additionally, it respects the management issues in the full life-cycle of the subscribed Grid services, e.g, inquiry, order, configuration, problem, quality, accounting and change management 10. By using CSM it is easier for a customer to identify failures and to receive more accurate information about his subscribed services. If needed, this also enables a Grid customer to provide a better quality of service to his own customers and users.

After the shift from computational to service Grids and many approaches to the management of distributed Grid resources as well as accompanying managability services (like for instance the Open Grid Service Infrastructure OGSI [11] and the Common Resource Model CRM [12]), the adaptation of stateful web services as a platform within the Open Grid Service Architecture OGSA 13. has brought new methods for the operation and management of integrated, distributed and manageable Grid environments. Furthermore, specifications like the WS-Resource Framework (to handle resource states), WS-Notification (to handle events), WSLA [14 respectively WS-Agreement (to handle service level agreements) or WS-Security (to handle authorization issues) have been invented and many of them are already used in current Grid middleware.

This situation introduces new opportunities as well as difficulties to adapt CSM mechanisms in Web and Grid service environments. Grid and Web service architectures equally drive a new paradigm of horizontal, inter-organizational and distributed deployments and provide various, distributed service access points at the 
sites of multiple providers. While on the other side, multiple customer organizations choose their endpoints from multiple providers and access them from different locations.

Grids are heterogenous, highly dynamic and loosely coupled environments that make use of virtualization concepts and introduced the concept of virtual organizations (VOs), whose management was e.g. discussed in [15], 16].

VOs as understood in this work, are dynamic collections of individuals, institutions and resources [17], established to achieve common business objectives. Providing customer oriented data and services to VOs is a new aspect to CSM and results in the challenge for CSM to support multiple virtual organizations.

\section{CSM in a Grid Monitoring and Accounting Scenario}

In our scenario, different scientific communities, organized as VOs, wish to use the german Grid infrastructure D-Grid to solve their computational problems on shared resources for computation. For the usage and management of these resources, the VO's members need to access monitoring and accounting data.

Our research focuses on the problem how this data can be provided from a Grid infrastructure to different Grid users that belong to VOs.

This involves the following questions:

- Which data is to be shared for the monitoring and accounting of Grid resources and their usage?

- How can the data be made available and accessible?

- Which data from a shared resource belongs to a given user or VO ?

- How can provider-centric data be mapped to the customers ?

- How can data about the usage, quality of services and the states of accompanying resources be retrieved by a user ?

- How can the data for Grid users be visualized in a dedicated way?

CSM as a concept is a useful approach to solve these questions, enabling the customer to retrieve and manage specific monitoring and accounting data.

Monitoring collects and provisions information about the usage, claim and affiliation of resources. Based on this information, accounting measures the accurate degrees of utilization (time, duration, host, user, provider, etc.) of the Grid resources and provides them to a billing component.

For Grid computing, monitoring and accounting of data is required with respect to different Grid users and their needs. A definition of a users roles, rights, subscribed services and accompanying resources is necessary. For example, 2] proposed different types of Grid users and a categorization as service end users, service providers and infrastructure system administrators. Methods to specify VOs as well as roles of their members and a specification of VO membership services can be found in [16]. For the scope of this paper, a VO membership service is assumed, which is able to provide data about the delegation of services and users to VOs. We use the CSM approach to customize the provisioned Grid services based on the information about the customers and their delegations. 
As illustrated in figure 1 for the example of Grid monitoring, accounting and billing, Grid service customers need monitoring and accounting information to determine whether they are getting the service levels they are paying for. CSM should allow the monitoring and controlling of service level agreements (SLAs) negotiated between providers and customers, in order to increase the perceived value of the services to the users as well as to lower the operational costs of service management for the service provider. This offers benefits for a services provider and also for its customer while respecting Service Level Agreements (which contain all agreements about the delivery of the service) and Service Level Specification (SLSs) (which is its technical description) 18. CSM should provide the necessary mechanisms to map the SLA and SLS settings onto the actual configuration of the service providers equipment.

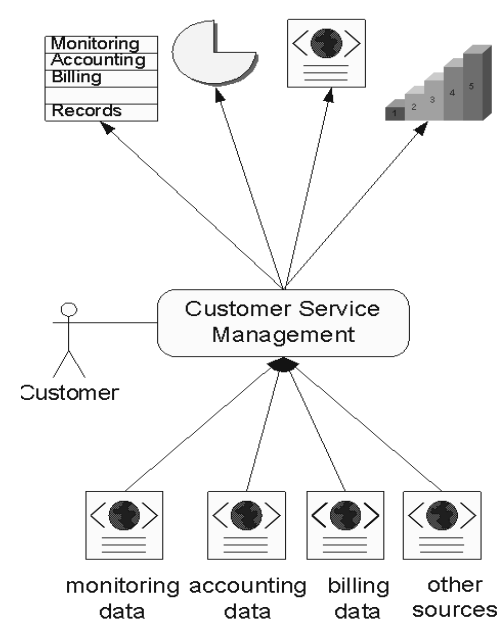

Fig. 1. CSM-based refinement of data

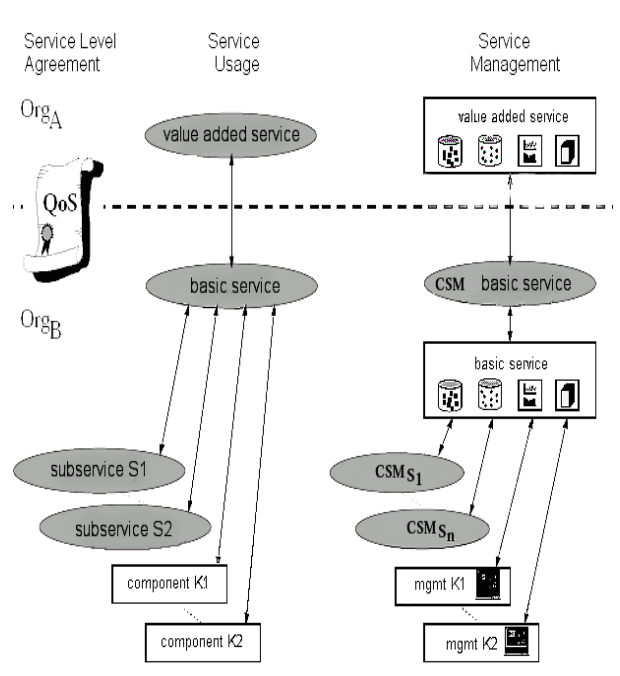

Fig. 2. A generic CSM scenario [1]

The provider does not have to provide all monitoring and accounting data to the end user who is not interested in the details of what is happening in underlying services (e.g. in the organization of the resource provider or the middleware). The user only needs information about the condition and the quality of the specific services he has subscribed to or which have been delegated to him.

Nevertheless, the customer and his users require information (and its visualization) in all phases of the lifecycle of the service provisioning:

- before service provisioning (e.g. procurement, signing a contract or a SLA)

- during service provisioning (e.g. control of the quality and states, failures, changes, maintenance times, statistics)

- after service provisioning (e.g. accounting, billing, optimization) 


\section{Architecture}

In the following, we first report about a generic concept for CSM as specified in [1. This concept points out different service types: At the provider side, there are basic services for the service management and their subservices as well as resources. The provider should also have a CSM basic service which provides logical views to the customer's side. At the customer side, there are value added services, representing the logical view produced by the CSM. While we discuss these service types in detail, we adapt every type to the specific situation in the Grid scenario. We further discuss aggregate levels in the distribution of metadata archives which provide - as subservices - the necessary data to the customer service management interface.

\subsection{The CSM Approach}

From a contractual point of view, as described in [1], customer and provider negotiate about QoS parameters which reflect the characteristics of the service. Based on these negotiations, a SLA is signed.

As shown in figure 2, CSM differentiates between the provider's and the customer's organization. This distinction enables the customer to find the best provider and service for the solution of a given problem.

For the provisioning of the service, the provider offers the service to his customer which can use the service itself or, in turn, can set up a value-added-service on top of it. For management purposes, the provider uses some kind of network and system management facilities (such as management platforms, trouble ticket systems and various other tools) that are necessary for the operation, administration, management and provisioning of the service. The customer will use similar facilities in his environment for the same aspects.

A Customer Service Management application is considered as a value added service in a customer's domain. It uses a basic CSM service at a CSM provider's domain. This service in turn uses services, sub-services and resources in its managed (service) network as shown in figure 2.

In a Grid environment, usually a Grid service provider $\left(\operatorname{Org}_{B}\right.$ in figure 2) puts Grid services on the disposal of different communities as customer organizations

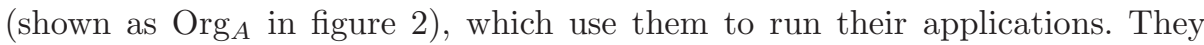
are organized as VOs. Thus, in a Grid, $\mathrm{Org}_{A}$ is a $\mathrm{VO}_{1 \ldots n}$ (see figure 3). On the provider side, there may be a basic organization $\operatorname{Org}_{B}\left(\mathrm{RO}_{\text {Gridserviceprovider in }}\right.$ figure 3) or, in the recursive case, a $\mathrm{VO}_{1 \ldots m}$.

Furthermore, the provider needs to gather data from subservices in his own organization as well as from external organizations. Thus, a provider's subservices can be provisioned by the provider itself, another provider in the role of an organization or a provider in the role of another VO.

\subsection{Value Added Management Services}

On the side of the customer's organization, a CSM application can act as a value added service, offering an interface to the users to manipulate and manage their basic services. 
In our Grid scenario, this means that the customer needs to receive available data from existing basic Grid monitoring and accounting services. This data should be already tailored to the context of the VO he is associated with. It is desired within a user-friendly interface that the data will be processed and visualized in a form wished by the user and /or the customer.

As the advancements in sciences and engineering put higher demands on tools for a high-performance large-scale visual data exploration and analysis, the interface should be designed to assist the customer in the use of appropriate visualization methods for his data sets.

The customer can also provide the functionality and information to his own customers as a value added service if there is no conflict of agreements.

\subsection{CSM Basic Service}

To be able to realize the value added service in the customer's domain, a CSM Basic Service must be provided at the CSM provider's network endpoint. The purpose of this service is to provide logical views for specific customers on the content of the provisioned services.

In 1 the CSM Basic Service is divided into two coupled services: An 'operative CSM provider' and an 'administrative CSM provider'. On the one hand, the 'operative CSM provider' is responsible for the technical and operational aspects, particularly the retrieval of customized and service-specific information as well as the provisioning of usage and management functionalities of the subscribed services. In addition, this information can comprehensively reflect SLA and QoS parameters fixed in contracts.

On the other hand, the 'administrative provider' is responsible for the administration of access rights (authorization) and the identification of customers (authentication). It also provides the definition, which service functionalities have been leased and how CSM information must be presented [1]. It should also include information about the provider's policies and the SLAs with the customers.

In a Grid environment, the information about which resources and services have been leased should be available as a part of a VO membership service [16. Using a coupling to such a service, a customized complex of services and subservices can be provisioned dynamically [19. Such complex services are able to provide a dedicated functionality which is needed by a given VO. In a CSM basic service which is coupled to a VO management, authorization, policies and SLAs as well as the required views to present data are dependent on the VO they are executed in. The usage as well as the management side of the service, authorization and authentication (AA), policies and SLAs as well as the usage and management of the presentations need to be VO dependent (see also figure 3).

Authorization and authentication may be realized by the use of a VO membership service or identity provider. Also, VO-specific Attribute Release Policies (ARP) may be defined to restrict the retrieval of data and functionality. The proposed architecture embeds and extends access control. 
But furthermore, it is necessary to realize functionalities like the handling of presentations and transformations, as well as policies and SLA issues dedicated to particular VOs. To describe how the data should be presented, VObased transformation rules are available from a VO dependent presentation management, e.g. provided as dedicated XSLT transformations. They can be processed by the presentation mapper of the CSM Basic Service or delivered to a customer's application, being processed there (as in the case of AJAX - Asynchronous Java script And XML).

The usage of the CSM

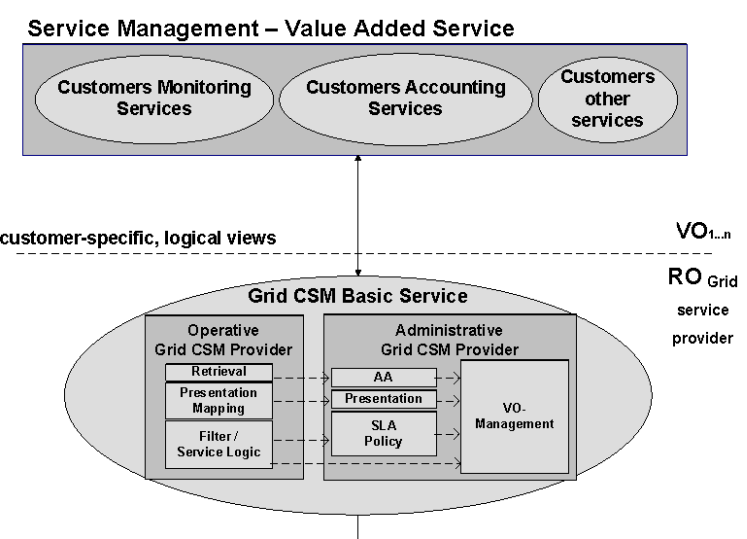

Service Management - Basic Service

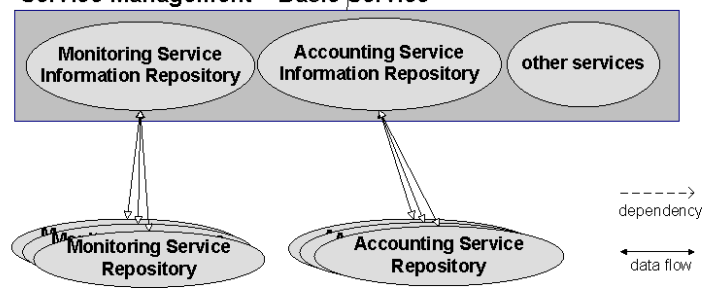

Fig. 3. The Grid CSM approach to data provisioning Basic Service at the 'operative provider' is determined by the VO based management information from the 'administrative provider': the possibility to retrieve data by the AA module; the presentation mapping by the presentation module and the set of data filtered and chosen by a service logic by the SLA, policy and VO management modules.

\subsection{Basic Services for Service Management}

'The basic services for Service Management represent arbitrary services in the IT environment [...] on the providers side' [1]. In fact, these services realize the functionalities which are provisioned to the customer. They can also offer management functionality for other services on the provider side. In a CSM scenario, these services are subservices of the CSM basic service, which acts as the interface to the customer.

In Grids, these services are often implemented on the basis of a service oriented architecture (SOA). It must be pointed out, that in a SOA based on web services also mechanisms such as WS-Security, WSLA 14 respectively WS-Agreement (for SLAs) and WS-Notification (for Events) are available, which can be used to implement the functionalities of a CSM basic service. Nevertheless, to collect information from the provider side, a customer should not be enforced to develop own applications to access accounting and monitoring information and a user interface is needed. 
In section 3, a basic service (or several distributed) for monitoring and accounting was considered. This service incorporates at least one database as a management information repository in which the information is collected. These services may be arbitrarily complex and consist of several subservices, e.g. local monitoring and accounting services and the accompanying sensors on the resources. They provide domain-specific metadata, e.g. in the form of XML files. For every organizational domain in a Grid environment (a traditional resource provider or a $\mathrm{VO}$ ), an information repository contains raw data for monitoring and accounting. This data is collected from multiple subservices and resources.

\subsection{Subservices and Resources}

The measured information is published through middleware using specific interfaces which depend on the used Grid service.

Basically, the monitoring and accounting subservices act as information repositories (or directories for mediation), recursively gathering their information from other repositories or - at the end of the chain - sensor services which implement the metering functionality.

For resources to be shared, sensors and repositories must be able to exchange basic monitoring, accounting as well as usage data in a common 20. and machine-readable format 21. For accounting, so called 'Usage Records' have been defined by the Open Grid Forum as a format for the exchange, as well as for the structured storage and utilization of accounting data in the Grid. They contain information about the use of resources and are based on a XML format. For monitoring, standardized information models like the OGF GLUE schema, CIM dialects or the Resource Description Framework RDF may be used.

\subsection{Aggregate Elements}

It is not only necessary that subservices and resources of the monitoring and accounting infrastructure are structured in an intelligent way so that they are able to hierarchically aggregate the correct information sources. The structure of the aggregated information retrieved from the subservices is also of importance. Thus, aggregate elements can be defined in the data, as done e.g. for accounting records in 22, where extended elements are introduced for the use across Grids and with heterogeneous extensibility. Such aggregate elements encapsulate a single aggregate accounting record. Elements of this kind also exist for monitoring services, bundling the information of multiple services in service groups (e.g., by using WS-ServiceGroups) or as database entries which contain aggregate data.

\subsection{Summary of the Grid CSM Approach}

Figure 3 summarizes the adapted CSM architecture as applied to the Grid monitoring and accounting scenario. Most importantly, in a Grid, the customer organization $\operatorname{Org}_{A}$ may be a $\mathrm{VO}_{1 \ldots n}$. A provider organization $R O$ inhabits the role of $\operatorname{Org}_{B}$ from the CSM scenario model. As pointed out in section 4.1), depending 
on the case, this provider organization $R O$ may also be a $\mathrm{VO}_{1 \ldots m}$, e.g. when a Customer Service Management between VOs is to be realized.

Between the layers of customer and provider, there is a mapping that is understood in terms of usage and management of the CSM basic service. It may be realized e.g. by providing protocols for the management of the presentation and transformation rules, the service level specifications, the policy rules, the identity providers and the VO management service.

Furthermore, a mapping between the Monitoring Service Information Repository as a Basic Service at the provider's site and the Monitoring Service Repositories as subservices can be perceived. It must be emphasized, that these repositories are provisioned by subservice providers, which are located in the local organization of the provider but also by subservice providers from remote ROs and VOs. The model may also be applied in a recursive way, gathering the data from another CSM Basic Service in a subproviders domain. This kind of stackability enables the sharing of data or functionality across different provider organizations which build different tools with CSM functionality.

\section{Implementation}

In the following, we report, how the introduced Grid components have been implemented to realize a CSM prototype with a monitoring and accounting functionality for the German D-Grid initiative, which is building a shared Grid infrastructure for many different scientific communities.

To satisfy a maximum of requirements in the D-Grid (see 23] and 24] while assuring a homogeneous management of basic services and underlying heterogenous resources from different resource providers, a SOA based on Globus Toolkit 4 was selected.

As the prototype was built from bottom up with a top down architecture in mind, the following sections begin with the description of the components used on resources, continue with the description of subservices, the basic services at the provider's side, the basic CSM service, and conclude with a CSM application providing the value added services.

\subsection{Basic Services, Subservices and Resources}

As a central underlying information system, the Monitoring and Discovery System MDS4 which is part of the Globus Toolkit 4, has been selected and installed in a testbed. In MDS4, sites and VOs may maintain one or more repositories to record available containers, resources, and services [25]. A distributed repository (implemented as a network of MDS4 DefaultIndexServices) was built, which gathers monitoring data from the resource providers in the Grid. For this purpose, a structure of repositories has been suggested, that introduces a central repository at every resource provider's site as well as a repository for every VO in the Grid, hosted on a machine that belongs to the VO. The data from the repositories at the different resource providers are brought together in the VO repositories by means of pushing with regular intervals. 
To measure status information about computing resources, standard MDS4 information providers have been used, placed on the local scheduling systems (e.g. Torque or SGE) at the Grid sites. To give the resource providers the opportunity to describe their sites, provide contact information, send maintenance messages and to advertise their geographical location into topological maps, an additional sensor has been created.

For accounting, a prototype was created based on components of SGAS (Swegrid Accounting System) 26. The prototype collects information about scheduled jobs and stores it in Resource Usage Records in the central database of a SGAS server 27.

\subsection{CSM Basic Service}

A tailored WebMDS system was installed to provide the presentation mapping functionality of a CSM basic service. WebMDS is an application for the Tomcat application server and provides logical views on the sites and computing resources on the raw data from the Grid by applying XSL-transformations, defined in the presentation management part of the architecture.

The setup is able to produce views on static VOs as they are specified by the trees connecting the metadata archives of the resource providers and their $\mathrm{VO}$ specific roots. At present time, all customers can select views on all existing VO specific information repositories.

The system is able to view the states of the computing resources and services of the VOs DGI, AstroGrid, MediGrid, C3-Grid, HEP, InGrid, TextGrid and Test at a website. The customer can select the VO he wishes to monitor on top of the webpage. To the left of the page, a presentation view on the selected VO's data can be chosen. Different XSLT transformations that generate HTML have been provided for this purpose, e.g. views on computing elements, clusters and workernodes, a drill-down view, a detailed service list, a XML-View, and a dynamic map of the geographic location of Grid sites as well as their maintenance status. For accounting, a view has been implemented, which is able to exhibit the most important data from accounting Usage Records such as CPU time, user host, provider host, start job time and end job time. For accounting, a view has been implemented, which is able to exhibit the most important data from accounting Usage Records such as CPU time, user host, provider host, start job time and end job time.

\subsection{CSM Application}

A usual web browser located in the domain of the customer can be used as a CSM application. HTML-GET Parameters are exchanged with the CSM basic service, defining the name of the repository and the kind of logical view the customer wants to see. This enables the customers to use web links given to them by the provider or to implement their own personal web sites or applications which retrieve the dynamic information from the CSM by using standard HTML queries. It is possible to receive XML documents in the style of a Representational State Transfer (REST), containing full or refined sets of resource 
metadata of a specific VO. The setup, including the XSLT-Transformations, can act as a basis for further integration of user interfaces in Grid Portals or as a basis for other applications, services or monitoring and accounting systems.

A quantitative experimental evaluation on the dynamic system performance characteristics of the MDS4 components central to the prototype has been presented by its developers in [25].

\section{Conclusions}

An architecture for the realization of Customer Service Management in a monitoring and accounting scenario was outlined. It is based on a CSM Basic service, a VO management service as well as additional modules for SLA, AA, presentation and policy management.

We presented an application of methods from CSM to the management and monitoring of Grid services to enable the refinement of granular customer and VO oriented views. A VO specific monitoring and accounting system for the productive Grid infrastructure relating to the D-Grid Integration Project was implemented. It was shown that the traditional models for CSM in principle still hold in Grid environments, but should be extended by a coupling of Grid services to VO management to support faster organizational dynamics as well as shorter lifecycles of customer organizations.

The concept can be applied to a broad scope of Grid services such as distributed metadata repositories or other areas which need a customer specific provisioning as e.g. parallel and distributed simulation services.

Further work is going to implement a CSM basic service that uses a dynamic VO membership service. It is also intended to integrate the repositories of additional VOs as well as necessary transformations to handle the corresponding VO specific data.

Acknowledgements. Parts of this work have been funded by the German Federal Ministry of Education and Research under contract 01 AK 800 B and by the EC IST-EMANICS Network of Excellence \#26854.

The authors wish to thank the members of the Munich Network Management (MNM) Team for helpful discussions and valuable comments on previous versions of this paper. The MNM Team founded by Prof. Dr. Heinz-Gerd Hegering is a group of researchers of the University of Munich, the Munich University of Technology, the University of Federal Armed Forces Munich and the Leibniz Supercomputing Centre of the Bavarian Academy of Sciences. Its web-server is located at http://www.mnm-team.org.

\section{References}

1. Langer, M.: Konzeption und Anwendung einer Customer Service Management Architektur (engl. Conception and Application of a Customer Service Management). $\mathrm{PhD}$ thesis, Technische Universität München (2001)

2. Norman, M.: Types of grid users and the Customer-Service Provider relationship: a future picture of grid use. In: Proceedings of the 2006 UK e-Science All Hands Meeting (2006) 
3. Sahai, A., Graupner, S., Machiraju, V., van Moorsel, A.: Specifying and Monitoring Guarantees in Commercial Grids through SLA. In: Proceedings of the 3rd IEEE International Symposium on Cluster Computing and the Grid, IEEE Computer Society Press, Los Alamitos (2003)

4. Chen, H., Jin, H., Mao, F., Wu, H.: Q-GSM: A QoS Oriented Grid Service Management Framework. In: Zhang, Y., Tanaka, K., Yu, J.X., Wang, S., Li, M. (eds.) APWeb 2005. LNCS, vol. 3399, Springer, Heidelberg (2005)

5. Magana, E., Serrat, J.: QoS Aware Policy-Based Management Architecture for Service Grids. In: Proceedings of the 14th IEEE International Workshops on Enabling Technologies, IEEE Computer Society Press, Los Alamitos (2005)

6. Bonnassieux, F., Harakaly, R., Primet, P.: The MapCenter Approach. In: Fernández Rivera, F., Bubak, M., Gómez Tato, A., Doallo, R. (eds.) Grid Computing. LNCS, vol. 2970, Springer, Heidelberg (2004)

7. Brent, N.C., Matthew, L.M., David, E.C.: The Ganglia Distributed Monitoring System: Design, Implementation,and Experience. In: Parallel Computing, vol. 30(7) (2004)

8. Legrand, I.C., Newman, H.B., Voicu, R., Cirstoiu, C., Grigoras, C., Toarta, M., Dobre, C.: MonALISA: An Agent Based, Dynamic Service System to Monitor, Control and Optimize Distributed Systems. In: CHEP 2004 (2004)

9. Langer, M., Loidl, S., Nerb, M.: Customer Service Management: Towards a Management Information Base for an IP Connectivity Service. In: Proceedings of the 4th IEEE Symposium on Computers and Communications, IEEE Computer Society Press, Los Alamitos (1999)

10. Nerb, M.: Customer Service Management als Basis für interorganisationales Dienstmanagement (engl. Customer Service Management as Basis for Interorganizational Service Management). PhD thesis, Technische Universität München (2001)

11. Tuecke, S., Czajkowski, K., Foster, I., Frey, J., Graham, S., Kesselman, C., Maquire, T., Sandholm, T., Snelling, D., Vanderbilt, P.: Open Grid Services Infrastructure (OGSI) Version 1.0. Technical report, Global Grid Forum (2003)

12. Stokes, E., Butler, N.: Common Resource Model (CRM). Technical report, Global Grid Forum (2003)

13. Foster, I., Kishimoto, H., Savva, A., Berry, D., Grimshaw, A., Horn, B., Maciel, F., Siebenlist, F., Subramaniam, R., Treadwell, J., Von Reich, J.: The Open Grid Services Architecture, Version 1.5. Technical report, Global Grid Forum (2006)

14. Keller, A., Ludwig, H.: Defining and Monitoring Service Level Agreements for dynamic e-Business. In: LISA 2002. Proceedings of the 16th USENIX System Administration Conference (2002)

15. Dreo Rodosek, G., Hegering, H.G., Stiller, B.: Dynamic Virtual Organizations as Enablers for Managed Invisible Grids. In: Proceedings of the 2006 IEEE/IFIP Network Operations and Management Symposium (NOMS), Vancouver, Canada (2006)

16. Schiffers, M.: Management dynamischer Virtueller Organisationen in Grids (engl.: Management of Dynamic Virtual Organizations in Grids). PhD thesis, LudwigMaximilians-Universität München (2007)

17. Foster, I., Kesselman, C., Tuecke, S.: The anatomy of the grid: Enabling scalable virtual organizations, vol. 15 (2001)

18. Betgé-Brezetz, S., Martinot, O., Delégue, G., Marilly, E.: Pro-Active SLA Assurance for Next Generation Network. In: WTC 2002. World Telecommunication Congress, Paris, France (2002) 
19. Baur, T., Bel Haj Saad, S.: Virtualizing Resources: Customer Oriented CrossDomain Monitoring for Service Grids. In: Proceedings of the 10th IFIP/IEEE Symposium on Integrated Management, IEEE Computer Society Press, Los Alamitos (2007)

20. Koo, M.y.: Accounting Interchange Natural Language Description (Requirements). Technical report, Global Grid Forum (2002)

21. Rodosek, G., Göhner, M., Golling, M., Kretzschmar, M.: Towards an Accounting System for Multi-Provider Grid Environments. In: IM 2007. Proceedings of the 10th IFIP/IEEE International Symposium on Integrated Management, IEEE Computer Society Press, Los Alamitos (2007)

22. Chen, X., Khan, A., Kant, D.: Aggregate Accounting Record Recommendation. Technical report, Global Grid Forum (2006)

23. gentschen Felde, N., Baur, T., Garschhammer, M., Reiser, H.: Anforderungen an das Monitoring, Ergebnisse aus den Erhebung bei den Communities und Ressourcenanbietern im D-Grid (engl.: Requirements for monitoring, results of a study of communities and resource providers). In: Rückemann, C.-P. (ed.): Ergebnisse der Studie und Anforderungsanalyse in den Fachgebieten Monitoring, Accounting, Billing bei den Communities und Ressourcenanbietern im D-Grid, Fachgebiete Monitoring, Accounting und Billing im D-Grid-Integrationsprojekt, pp. 45-63 (2006)

24. Rückemann, C.P., Göhner, M.: Anforderungen an das Accounting (engl.: Requirements for accounting). Technical report, D-Grid (2006)

25. Schopf, J., Raicu, I., Perlman, L., Miller, N., Kesselman, C., Foster, I., D'Arcy, M.: Monitoring and Discovery in a Web Services Framework: Functionality and Performance of Globus Toolkit MDS4. In: Proceedings of the 15th IEEE International Symposium on High Performance Distributed Computing, IEEE Computer Society Press, Los Alamitos (2006)

26. Elmroth, E., Gardfjell, P., Mulmo, O., Sandholm, T.: An OGSA-Based Bank Service for Grid Accounting Systems. In: Applied Parallel Computing. State-of-the-art in Scientific Computing, Springer, Heidelberg (2004)

27. Baur, T., Bel Haj Saad, S., Göhner, M.: Customer Service Management für Grid Monitoring und Accounting (engl.: Customer Service Management for Grid monitoring and accounting). Technical report, D-Grid (2007) 